\title{
UNA NUEVA ESPECIE DE ILEX L. (AQUIFOLIACEAE), DEL NORESTE DEL ESTADO DE QUERÉTARO, MÉXICO*
}

\author{
Eleazar Carranza GonzÁlez \\ Instituto de Ecología, A.C., Centro Regional del Bajío, Apdo. postal 386, \\ 61600 Pátzcuaro, Michoacán, México
}

\section{RESUMEN}

Se describe como especie nueva a Ilex servinii (Aquifoliaceae) del estado de Querétaro, México. Este árbol presenta hojas más anchas que las otras especies del centro del país, asimismo, sus flores son más grandes y es prácticamente glabro. Se encontró en bosques de pino-encino y mesófilo de montaña del noreste de dicho estado y al parecer es muy escaso. Se hace una comparación del nuevo taxon con especies con las que puede confundirse y que crecen en el centro de México, como son I. tolucana Hemsl., I. discolor Hemsl. e I. brandegeana Loes.

Palabras clave: Aquifoliaceae, Ilex, México, Querétaro.

\begin{abstract}
Ilex servinii (Aquifoliaceae) a new species from the northeastern portions of the state of Queretaro, Mexico, is described and illustrated. This tree presents wider leaves than the other species of central Mexico, its flowers are bigger and it is practically glabrous. It occurs in oak-pine forest and cloud forest. The species is very rare. A comparison is made between Ilex servinii and I. tolucana Hemsl., I. discolor Hemsl., I. brandegeana Loes., three other species from central Mexico.
\end{abstract}

Key words: Aquifoliaceae, Ilex, Mexico, Queretaro.

\footnotetext{
* Trabajo realizado con apoyo económico del Instituto de Ecología, A. C. (cuenta 902-07), del Consejo Nacional de Ciencia y Tecnología y de la Comisión Nacional para el Conocimiento y Uso de la Biodiversidad.
} 


\section{INTRODUCCIÓN}

El género Ilex L., el único de la familia Aquifoliaceae, está conformado por árboles o arbustos por lo general dioicos, que habitan en zonas tropicales y templadas de ambos hemisferios, principalmente en Centro y Sudamérica, donde se presenta su centro de diversificación (Lawrence, 1951). A nivel mundial se calcula en total unas 500 especies (o hasta 650, de acuerdo con Edwin, 1967) y para México se desconoce el número de ellas; sin embargo, en diferentes trabajos en los que se mencionan estas plantas para el territorio mexicano, se enlistan alrededor de 11 (Standley, 1923; Standley y Steyermark, 1949; González, 2000; Calderón de Rzedowski, 2001). También se han observado en algunas de las colecciones importantes del país otros tres o cuatro taxa, cuyas determinaciones al parecer son correctas, pero no corresponden a los nombres citados para el país. Asimismo, en dichos acervos, se encuentran otros ejemplares que pueden representar hallazgos novedosos. Por todo lo anterior es importante y necesario hacer la revisión de dicho género en México.

En la región noreste del estado de Querétaro, cerca de la frontera con el de San Luis Potosí, se localizaron algunas muestras de este género que difieren de todas las especies conocidas. No se encontraron individuos parecidos en los herbarios mexicanos ENCB, IEB y MEXU, por lo que se llegó a la conclusión de que se trataba de una especie nueva. El material se obtuvo en 1990 y, desde hace unos cuatro años, se ha tratado en varias ocasiones de localizar los árboles para contar con individuos femeninos en flor y elaborar una descripción completa. Aunque no se tuvo éxito en esta búsqueda, se decidió proponer el nuevo taxon, esperando que en un futuro próximo se complemente la descripción.

Ilex servinii E. Carranza sp. nov. Fig. 1.

Arbor ad $10 \mathrm{~m}$ alta; petioli 1.3-2.2 cm longi, glabri, folia elliptica vel oblongoelliptica, $6.5-12 \mathrm{~cm}$ longa, 3-6.5 cm lata, obtusa usque acuminata, rare retusa, basi acuta usque rotundata, margine integerrima, revoluta, glabra; inflorescentiae fasciculatae vel breviter paniculatae, pedunculi ad $1 \mathrm{~cm}$ longi; flores masculini 8-12 mm diametro, petala oblonga, 3.5-5.5 mm longa, albida; filamenta 2-3 mm longa, antherae 1.5-2 mm longae, subglobosae; drupa 4-6 mm diametro, glabra, rufa vel rufo-flavescens; semina 4-5, triquetra, 3-5 $\mathrm{mm}$ longa.

Árbol de 6 a 10 m de alto; ramillas glabras; hojas alternas, estípulas largamente deltoideas, de alrededor de $1 \mathrm{~mm}$ de largo, glabras, caedizas, pecíolo de 1.3 a $2.2 \mathrm{~cm}$ de largo, glabro, lámina elíptica u oblongo-elíptica, concolora, de 6.5 a $12 \mathrm{~cm}$ de largo, de 3 a $6.5 \mathrm{~cm}$ de ancho, ápice obtuso a acuminado, rara vez retuso, base aguda 
Carranza: Una nueva especie de Ilex de Querétaro

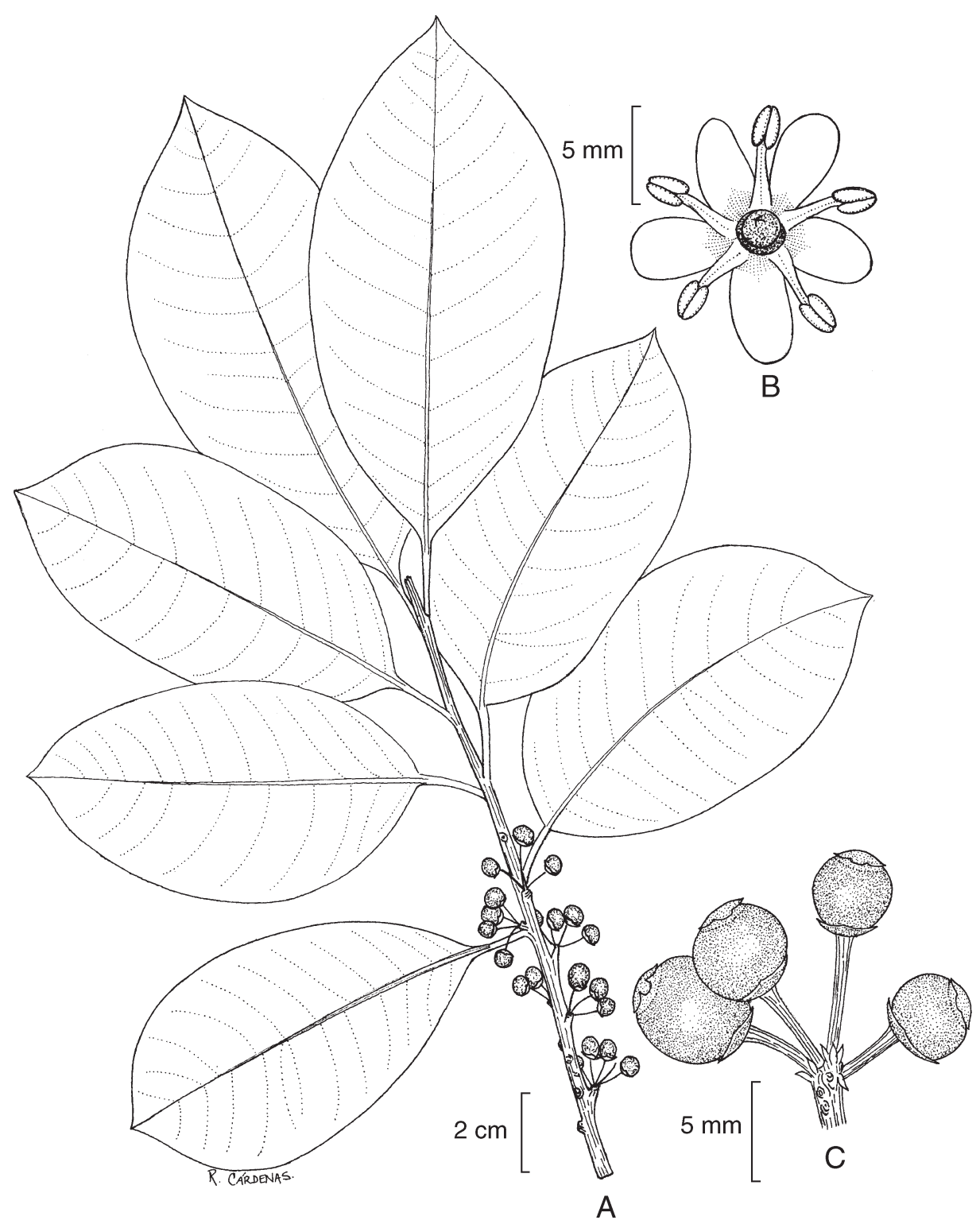

Fig. 1. Ilex servinii. A. Rama con frutos; B. Flor masculina; C. Frutos. Ilustrado por Rogelio Cárdenas. 
a redondeada, margen entero o a veces con 1 a 3(4) dientecillos a cada lado en los primeros 3 a $6 \mathrm{~mm}$ a partir de la base de la lámina, el resto finamente revoluto, la vena principal muy elevada en el envés, un poco hundida en el haz, las venas secundarias inconspicuas o apenas notorias en ambas superficies, algo coriácea, glabra o a veces con algunos pelos en la nervadura principal, sobre todo por el envés; inflorescencias de ambos sexos fasciculadas, a veces cortamente paniculadas, regularmente con 3 a 9 flores; brácteas subuladas, agudas, de 1 a $3 \mathrm{~mm}$ de largo, algo pilosas, pedúnculo (de existir) de alrededor de 8 a $10 \mathrm{~mm}$ de largo, de unos $2 \mathrm{~mm}$ de grueso, glabro, rugoso, acanalado o estriado; pedicelos de las flores masculinas de (2)4 a $8 \mathrm{~mm}$ de largo; flor masculina de unos 8 a $12 \mathrm{~mm}$ de diámetro, sépalos más o menos deltoideos, unidos en la base en alrededor de $0.7 \mathrm{~mm}$, de 1.5 a $2 \mathrm{~mm}$ de largo, agudos a obtusos, margen algo crenado, glabros; pétalos blanquecinos, oblongos, redondeados en el ápice, un poco más angostos hacia la base, de 3.5 a $5.5 \mathrm{~mm}$ de largo, de 2 a $2.7 \mathrm{~mm}$ de ancho; estambres alternos con los pétalos, filamentos de 2 a $3 \mathrm{~mm}$ de largo, más o menos aplanados, anteras subglobosas a ovadas, de 1.5 a $2 \mathrm{~mm}$ de largo, tecas abriendo por hendiduras longitudinles; flores femeninas desconocidas; fruto drupáceo, en grupos de hasta 8 o solitarios, de 4 a $6 \mathrm{~mm}$ de diámetro, rojo o rojo-amarillento, glabro, con 4 o 5 pirenos; pireno (endocarpo que cubre a la semilla) subtrígono, con 1 semilla de 3 a $5 \mathrm{~mm}$ de largo.

Tipo: México, Querétaro, municipio de Jalpan, 4-5 km al oriente de La Parada (Valle Verde), bosque húmedo de pino-encino, alt. $1400 \mathrm{~m}, 24 . \mathrm{IV} .1990$ (individuo masculino con flores), B. Servín 150 (holotipo: IEB; isotipos: ENCB y MEXU).

Material adicional revisado: Querétaro, municipio de Jalpan, 5-6 km al oriente de La Parada (Valle Verde), 29.III.1990 (individuo masculino con botones florales), B. Servín 74 (IEB); 3-4 km al oriente de La Parada (Valle Verde), 06. IX.1990 (individuo femenino con frutos), B. Servín 469 (IEB).

Distribución: Se conoce únicamente del noreste del estado de Querétaro, en una zona muy próxima a la frontera con el de San Luis Potosí.

Hábitat: Este árbol crece en forma muy escasa en lugares protegidos, húmedos, con bosques de pino-encino y mesófilo de montaña. Algunas de las especies arbóreas que con mayor frecuencia se observan en el área son: Liquidambar styraciflua, Pinus greggii, Quercus affinis, Q. germana, Rhamnus capraeifolia var. capraeifolia, Ternstroemia sylvatica, Turpinia occidentalis, entre otras. La altitud en que se ha registrado oscila entre 1300 y 1500 m. 
Fenología: Se le encontró en flor durante marzo y abril y con frutos en septiembre. No se hallaron flores femeninas.

Etimología: El epíteto específico se dedica al Sr. Benito Servín Orozco, quien obtuvo los ejemplares conocidos, además de ser un colector destacado para el proyecto Flora del Bajío en la región noreste del estado de Querétaro.

Ilex servinii se diferencia en general de las otras especies del género presentes en el centro de México, por sus hojas más anchas y con los márgenes enteros o casi enteros, así como por sus flores masculinas más grandes y la ausencia casi total de indumento (Cuadro 1). Las inflorescencias fasciculadas recuerdan las de I. tolucana Hemsl., sin embargo, esta última sólo desarrolla flores con este tipo de arreglo, sus hojas alcanzan hasta $4.5 \mathrm{~cm}$ de ancho con el margen crenadoserrulado, los sépalos miden de $1 \mathrm{a} 1.5 \mathrm{~mm}$ de largo, los pétalos son ampliamente ovados, de 2 a $4 \mathrm{~mm}$ de largo, mientras que la primera también tiene flores reunidas en forma de panícula, las hojas son de hasta $6.5 \mathrm{~cm}$ de ancho y con el margen entero (a veces con algunos dientes diminutos), sépalos de 1.5 a $2.5 \mathrm{~mm}$ de largo y pétalos obovados a oblongos, de 3.5 a $5.5 \mathrm{~mm}$ de largo. El margen entero también lo tiene I. dugesii Fernald (Rose, 1895; González, 2000), que llega a presentar en ocasiones algunos dientes, pero el envés de las hojas es amarillento-tomentoso, lo que las hace bicoloras, y los frutos son solitarios, por su parte las hojas del nuevo taxon son más bien concoloras y los frutos están agrupados. En el caso de I. brandegeana Loes., que tiene flores casi del mismo tamaño que $I$. servinii, la diferencia principal radica en el margen de las hojas que es prácticamente entero en ésta y serrulado-dentado con aristas en la primera.

Clave para separar las especies más relacionadas con I. servinii en el centro de México

1. Margen de las hojas con dientes aristados, rara vez sin aristas; venación muy aparente (protuberante) en el envés I. brandegeana 1. Margen de las hojas entero, crenado-serrulado a serrado (rara vez aristado en I. tolucana); venación no aparente en el envés.

2. Frutos solitarios; ramillas y hojas densamente amarillento-tomentosas

I. dugesii

2. Frutos agrupados en cimas o fascículos, en ocasiones algunos solitarios en la misma ramilla; ramillas no densamente amarillento-tomentosas.

3. Hojas discoloras (el envés más pálido que el haz), de 2 a 4.5(6) cm de largo, de 1 a 2.5(4) $\mathrm{cm}$ de ancho, obovadas, elíptico-oblongas a elípticolanceoladas; flor tetrámera I. discolor 


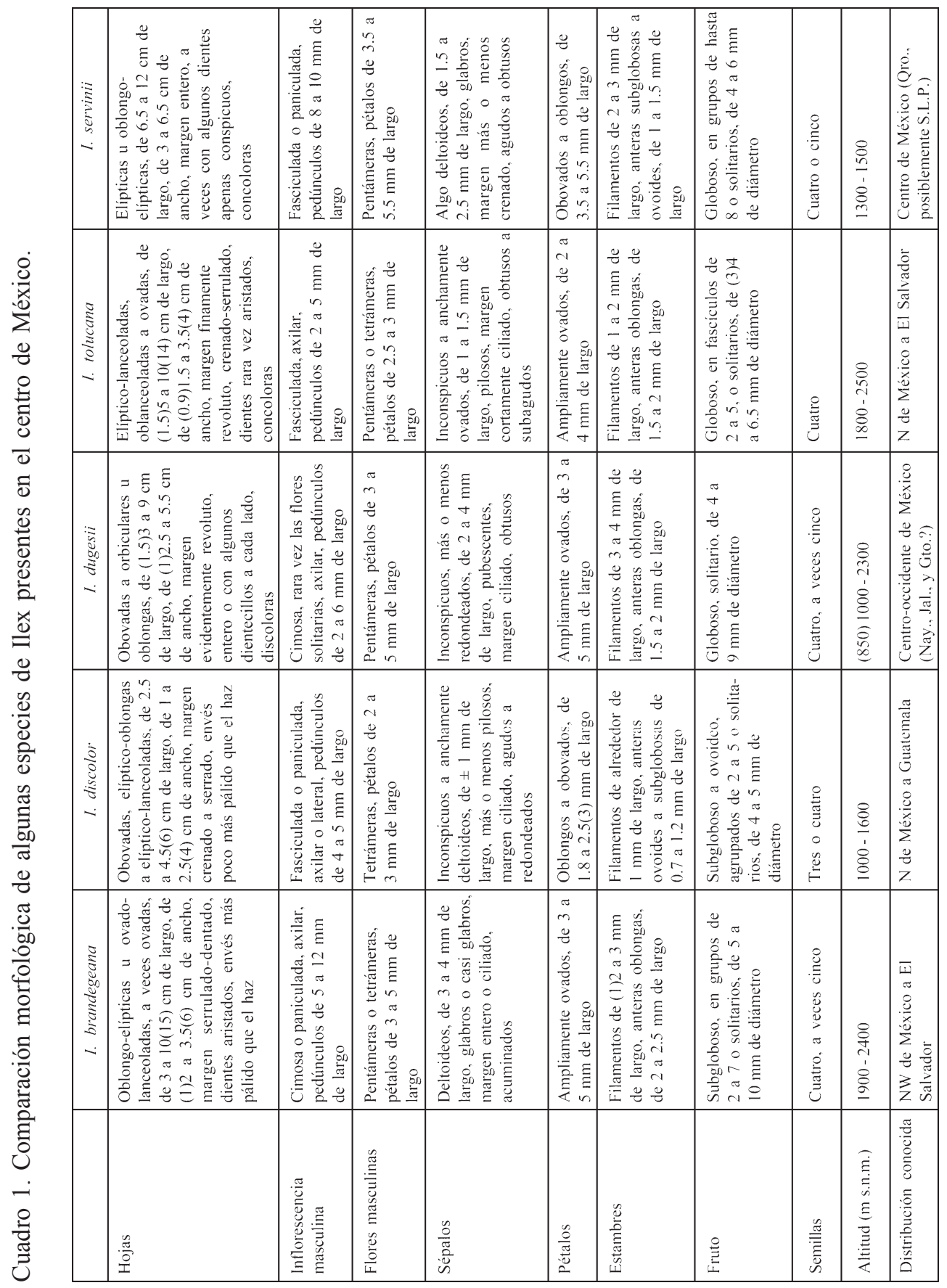


3. Hojas concoloras, de (4) 5 a $12 \mathrm{~cm}$ de largo, de (1.5) 3 a $6.5 \mathrm{~cm}$ de ancho, elípticas, oblongo-elípticas, elíptico-lanceoladas, oblanceoladas a ovadas; flor tetrámera o pentámera.

4. Pétalos de las flores masculinas de 3.5 a $5.5 \mathrm{~mm}$ de largo; margen de las hojas dentado en los primeros 3 a $6 \mathrm{~mm}$ a partir de la base, entero o raras veces con dientes incospicuos y ligeramente revoluto en el resto; planta conocida sólo del extremo NE de Querétaro ........... I. servinii

4. Pétalos de las flores masculinas de 2.5 a $3 \mathrm{~mm}$ de largo; margen de las hojas serrulado o crenado serrulado, los dientes a veces cortamente aristados; planta con distribución conocida amplia (desde el N de México hasta El Salvador) …......................................................... I. tolucana

\section{AGRADECIMIENTOS}

Le doy las gracias al Sr. Benito Servín, quien amablemente dedicó mucho de su tiempo para localizar árboles de esta especie en los últimos años. A Arnulfo Blanco, Ivonne Silva, Carlos Silva y Benjamín Magaña, por su compañía en las diferentes exploraciones en busca de los mismos. También a Rogelio Cárdenas la elaboración de la figura, así como a los Drs. Jerzy Rzedowski y Victor Steinmann la lectura crítica del manuscrito y a los revisores anónimos, cuyas sugerencias fueron de gran valía para ver culminado este trabajo.

\section{LITERATURA CITADA}

González, L. M. 2000. La familia Aquifoliaceae en el estado de Jalisco, México. Colección Flora de Jalisco. Vol. 6. Instituto de Botánica. Universidad de Guadalajara. Guadalajara. 27 pp.

Calderón de Rzedowski, G. 2001. Aquifoliaceae. In: Rzedowski, G. C. de, J. Rzedowski y colaboradores. 2001. Flora fanerogámica del Valle de México. 2a ed., Instituto de Ecología, A.C. y Comisión Nacional para el Conocimiento y Uso de la Biodiversidad, Pátzcuaro. $1406 \mathrm{pp}$.

Edwin, G. 1967. Aquifoliaceae. In: Flora of Panama. Ann. Missouri Bot. Gard. 54:381.

Lawrence, G. H. 1951. Taxonomy of vascular plants. The Macmillan Company. Nueva York. p. 576.

Rose, J. N. 1895. Ilex dugesii, n. sp. Bot. Gaz. 20: 533.

Standley, P. C. 1923. Aquifoliaceae. In: Trees and shrubs of Mexico. Contr. U. S. Nat. Herb. 23(3): 673-676. 
Acta Botanica Mexicana 69: 133-140 (2004)

Standley, P. C. y J. Steyermark. 1949. Ilex. In: Flora of Guatemala. Filediana, Bot. 24(6): 197201.

Recibido en septiembre de 2004.

Aceptado en noviembre de 2004. 